\title{
Systematic study of the decay rates of antiprotonic helium states
}

\author{
H. Yamaguchi,* R. S. Hayano, T. Ishikawa, J. Sakaguchi, and E. Widmann \\ Department of Physics, University of Tokyo, 7-3-1 Hongo, Bunkyo-ku, Tokyo 113-0033, Japan \\ J. Eades and M. Hori \\ CERN, CH-1211 Geneva 23, Switzerland \\ H. A. Torii \\ Institute of Physics, University of Tokyo, Komaba, Meguro-ku, Tokyo 153-8902, Japan \\ B. Juhász \\ Institute of Nuclear Research of the Hungarian Academy of Sciences, H-4001 Debrecen, Hungary \\ D. Horváth \\ KFKI Research Institute For Particle and Nuclear Physics, H-1525 Budapest, Hungary \\ T. Yamazaki \\ RI Beam Science Laboratory, RIKEN, Wako, Saitama 351-0198, Japan \\ (Received 19 January 2004; published 8 July 2004)
}

\begin{abstract}
A systematic study of the decay rates of antiprotonic helium $\left(\bar{p}^{4} \mathrm{He}^{+}\right.$and $\left.\bar{p}^{3} \mathrm{He}^{+}\right)$at CERN Antiproton Decelerator has been made by a laser spectroscopic method. The decay rates of some of its short-lived states, namely those for which the Auger rates $\gamma_{A}$ are much larger than their radiative decay rates $\left(\gamma_{\mathrm{rad}} \sim 1 \mu s^{-1}\right)$, were determined from the time distributions of the antiproton annihilation signals induced by laser beams, and the widths of the atomic resonance lines. The magnitude of the decay rates, especially their relation with the transition multipolarity, is discussed and compared with theoretical calculations.
\end{abstract}

DOI: 10.1103/PhysRevA.70.012501

PACS number(s): 36.10.-k, 32.80.Dz

\section{INTRODUCTION}

The antiprotonic helium atom $\left(\bar{p} \mathrm{He}^{+}\right)$is an exotic threebody system consisting of an antiproton, an electron, and a helium nucleus [1-3]. Detailed studies have been done for its metastable-state region, where the principal quantum number $n$ and angular momentum $l$ are both around 38, and where lifetimes against annihilation can be as long as several microseconds. Recently stringent $C P T$-violation limits on the antiproton mass and charge were determined [4] at CERN Antiproton Decelerator using a laser spectroscopic method to measure some transition energies of the $\bar{p} \mathrm{He}^{+}$atom to a precision of 50-200 ppb. The measured transition energies were compared with two independent variational three-body calculations with QED corrections by Korobov [5-7] and by Kino [8,9].

In addition to their level energies $E_{r}$, an important property of the states of this exotic atom is their decay rate $\gamma$, which forms the imaginary part of the complex energy

$$
E=E_{r}-i \frac{\gamma / 2 \pi}{2}
$$

In particular, the Auger rates $\gamma_{A}$, which are dominant in the total decay rate for some $\bar{p} \mathrm{He}^{+}$states, have been calculated

\footnotetext{
*Present address: Center for Nuclear Study (CNS), University of Tokyo, RIKEN Campus, 2-1 Hirosawa, Wako, Saitama 351-0198, Japan.
}

by many theorists [10-13] over a period of more than 30 years. The latest calculations with complex coordinate rotation (CCR) method by Korobov [7] and Kino [9] yield both real and imaginary parts of the complex energy at the same time, and the both parts are expected to have absolute precisions of the same order. An independent check on the validity of these three-body calculations can therefore be obtained by measuring the decay rates of Auger-dominant states.

Although the $C P T$-violation limits [4] were obtained by the level-energy measurements with high relative precisions of 50-200 ppb, discussions of the decay rates with such high accuracy are difficult both experimentally and theoretically. The experimental difficulty originates in the finite response time in the detection of the antiproton annihilations, and in the broadening of the atomic spectral linewidth from various sources (laser linewidth, Doppler width, and so on). Theoretically, the difficulty lies in representing continuumcoupled discrete states with a limited set of wave functions, and in the small magnitude of the Auger widths (order of megahertz-gigahertz) compared to the transition energies (0.4-1 PHz), from visible light to ultraviolet. However, due to the smallness of the Auger widths, the absolute precisions of both experimental and theoretical decay rates can be as good as those of the level energies for some states, and their measurements would be meaningful for the verification of the CCR calculations.

A prominent and important feature of the theoretically calculated Auger rates is their drastic dependence on the lowest possible transition multipolarity $L$, which is equal to the 
minimum angular momentum carried away by the Auger electron $[10,12]$. The theoretical rates are approximated by a rough estimation

$$
\gamma_{A} \sim 10^{17-3 L} \mathrm{~s}^{-1} \text { for } L=2,3,4 .
$$

States with $L \leqslant 3$ have radiative rates of $10^{5}-10^{6} \mathrm{~s}^{-1}$. They are consequently Auger dominated, and we refer to them as "short-lived" states. For states $L>3$, fast Auger decays are suppressed, and the atom deexcites only radiatively. These are the states which we call "metastable."

By 2001, we had studied the decay rates of eight $\bar{p}^{4} \mathrm{He}^{+}$ states $[14,15]$. Although most of them have decay rates in accordance with the above approximate Eq. (2), we found two exceptions. One was the $(n, l)=(37,33)$ state, which is possibly affected by an electron-excited configuration $(32,31)_{\bar{p}} \otimes(3 d)_{e}$ having a nearby level energy [11]. Another was the $(32,31)$ state, the decay rate of which our lowdensity measurement with the RFQ decelerator $[4,16]$ revealed to be greatly enhanced by collisions. We call these decay-rate discrepancies "anomalies" [15].

\section{MEASUREMENT OF DECAY RATES}

In 2001-2003, we studied decay rates of ten states of $\bar{p} \mathrm{He}^{+}$, including seven states of $\bar{p}^{3} \mathrm{He}^{+}$. A detailed description of the experimental procedure using laser spectroscopy can be found in Refs. [17-19].

Antiproton pulses of duration $\sim 300 \mathrm{~ns}$, each of which contained some $10^{7}$ particles were brought to rest in a helium gas target at a temperature of $5.5 \mathrm{~K}$ and pressures between 20 and $200 \mathrm{kPa}$. Charged pions produced by their subsequent annihilation were detected by Čerenkov counters, annihilation time spectra produced by these pions being recorded as analog pulses by a digital oscilloscope [20].

When we applied a laser pulse tuned to a wavelength of a metastable to short-lived transition, a sharp peak appeared in the delayed annihilation time spectrum, the intensity (area) of which was proportional to the number of antiproton annihilations induced by the laser pulse. Spectral line profiles could then be obtained by plotting the peak intensity against the laser wavelength as this was scanned through the central transition wavelength.

Three methods were used to deduce decay rates according to their magnitude.

\section{A. Decay rates faster than $6 \mathrm{~ns}^{-1}$ (wide resonances)}

When the decay rate is fast enough, a transition to the state is observed as a wide resonance having a natural width larger than the laser linewidth $(\sim 1 \mathrm{GHz}$ at "high resolution" mode, see later). Figure 1 shows four $(n, l) \rightarrow(n+1, l-1)$ type resonances newly measured in this work. Such transitions are called "unfavored", since they have typically ten times smaller transition dipole moments than $(n, l) \rightarrow(n$ $-1, l-1$ ) (or "favored") transitions. As mentioned earlier, we may observe collisionally enhanced decay rates, not the pure Auger rates themselves, with this method. However, the measurements were performed at a specific target atomic density of $3 \times 10^{20} \mathrm{~cm}^{-3}(6 \mathrm{~K}, 20-30 \mathrm{kPa})$ for these four

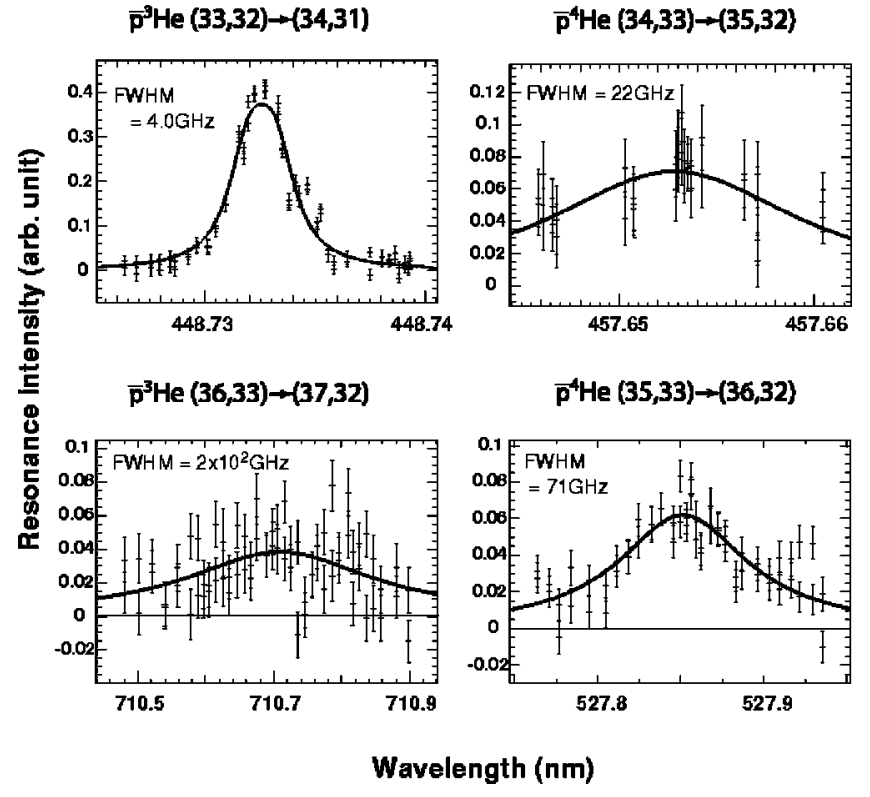

FIG. 1. Wide resonances scanned for the Auger rate deduction. Their full width at half maximum was obtained by fitting with Lorentz functions. Statistical errors are shown.

states, since for most states $([14,15])$ no systematic density variation of the decay rates larger than the experimental error was observed.

The dye laser used in our laser system (Lambda Physik Inc. Scanmate 2E) could be operated in "broadband" or "high resolution" modes. In the broadband mode, the power was high (a few $10 \mathrm{~mJ} / \mathrm{shot}$ ) but the linewidth was several gigahertz, while in the high resolution mode, the laser frequency linewidth was severely constrained by an intracavity etalon to about $1 \mathrm{GHz}$. For some of the unfavored transitions shown in Fig. 1, the laser was operated in the broadband mode to fulfill the requirement of high power for the unfavored resonances. It is difficult to know the exact linewidth during the resonance scans. By investigating the spectra broadening measured by Fizeau interferometers, the linewidth could be determined as $4-10 \mathrm{GHz}$, except for the $448 \mathrm{~nm}$ case for which the laser was operated in the high resolution mode with a linewidth of $0.7-1.3 \mathrm{GHz}$.

The analysis procedure for each of these states was the following. The resonance spectrum was fitted with two identical Voigt functions (convolutions of a Gaussian and a Lorentzian), separated by a fixed frequency splitting. This splitting was introduced to represent the hyperfine splitting [21,22], caused by the coupling of the electron spin and the $\bar{p}$ orbital magnetic moment. For the hyperfine splitting, theoretical values by Bakalov and Korobov [23] were used, or $(2 \pm 0.5) \mathrm{GHz}$ assumed for uncalculated states. The width of the Gaussian component was fixed at the laser linewidth plus Doppler broadening, and the Lorentzian width was obtained from the fitting procedure. The Lorentzian width was considered to be approximately equal to the natural width $\Delta \nu_{\text {nat }}$ because the other factors such as the collisional and power broadening were estimated to be much smaller than $1 \mathrm{GHz}$. Finally, $\Delta \nu_{\text {nat }}$ was converted to the decay rates $\gamma$ by 

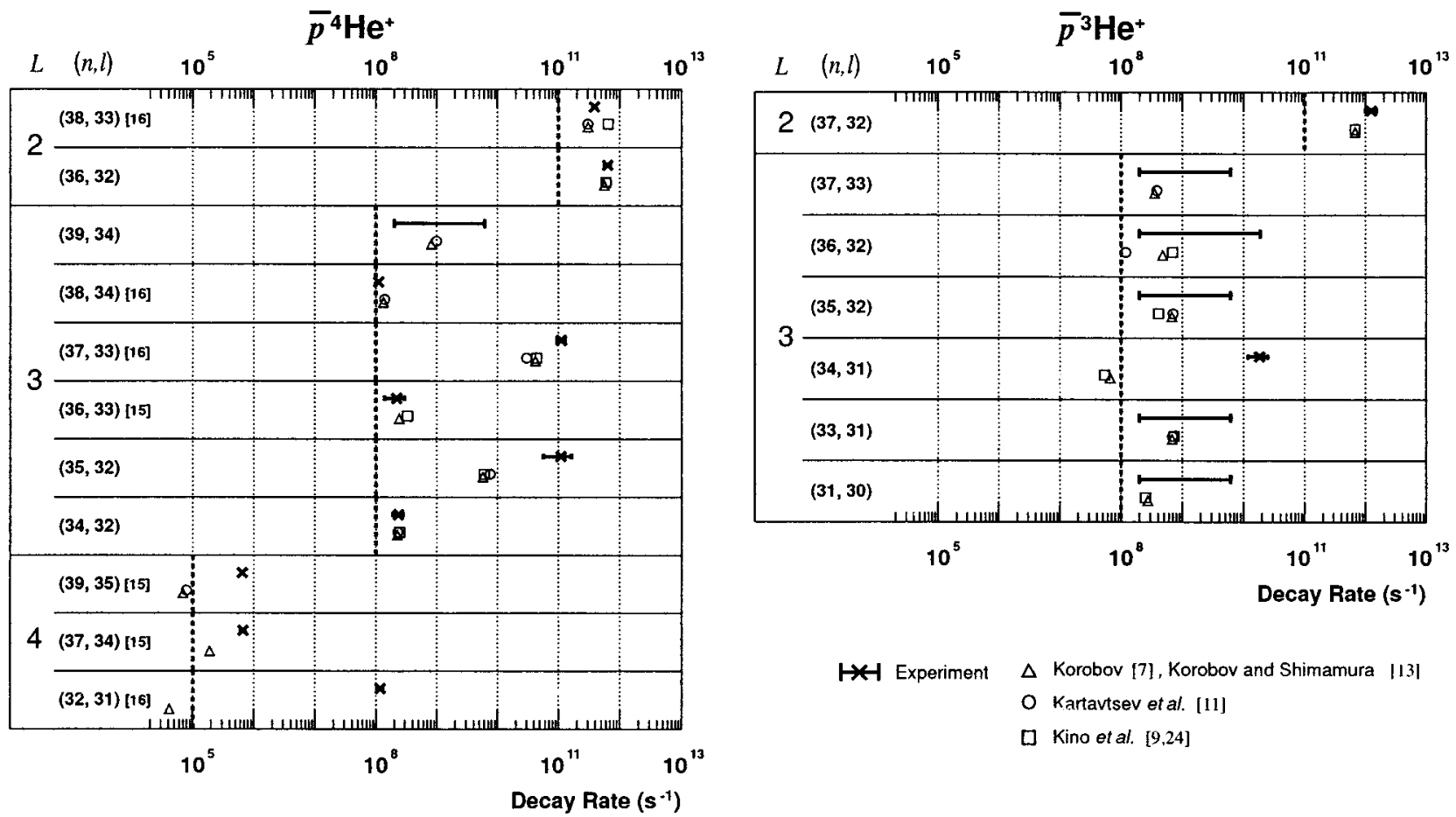

FIG. 2. Experiment-theory comparison of the decay rate in logarithmic scale. All the data including past measurements [14,15] are shown. The bold vertical dotted lines show the typical Auger rates estimated by Eq. (2). The experimental points without crossed markers show that the rates are between two experimental limits, and not directly determined (see the case of Sec. II C). Some of the experimental error bars are too small to see.

$$
\gamma=2 \pi \Delta \nu_{\text {nat }}
$$

The errors were estimated mainly from the statistical fluctuations, uncertainty of the laser linewidths and hyperfine splittings.

\section{B. Decay rates of about $0.2 \mathrm{~ns}^{-1}$ or slower (long tail peaks)}

If the decay rates are slow enough, antiprotons which annihilate from the Auger-dominated state do so after a measurable additional delay. We can then obtain the decay rates directly by fitting the "tails" of the laser resonance peaks with exponential functions. The effect of the fall time of the Hamamatsu Photonics R5505GX-ASSYII photomultiplier tubes (about $4 \mathrm{~ns}$ ) [20] in prolonging these tails was corrected for in the fitting procedure. This method is applicable for decay rates up to about $0.1-0.2 \mathrm{~ns}^{-1}$, depending on the performance of the photomultiplier tubes.

In this work, only the $(34,32)$ state of $\bar{p}^{4} \mathrm{He}^{+}$could be considered to fall in this category. This state was measured also in the previous work [15]; in the present experiments we obtained a more reliable result by using a fast-response photomultiplier tube.

The rate was measured at three different atomic density points in $0.2-2 \times 10^{21} \mathrm{~cm}^{-3}$, and no significant differences were found between those results.

\section{Intermediate rates}

For the states that do not show either wide resonances or prolongation of laser peaks, we cannot measure their decay

rates directly. The daughter states of an unfavored [the $(35,33) \rightarrow(36,32)$ of $\bar{p}^{3} \mathrm{He}^{+}$] and five favored resonances [to the $(37,33),(35,32),(33,31)$, and $(31,30)$ of $\bar{p}^{3} \mathrm{He}^{+}$, and the $(39,34)$ of $\bar{p}^{4} \mathrm{He}^{+}$from each corresponding $(n+1, l+1)$ state] come under this category. In this case, we concluded that their decay rates are between the two experimental limits. The lower limit $\left(0.1 \mathrm{~ns}^{-1}\right)$ is determined mainly by the instrumental prolongation effect of the laser peak, as mentioned earlier. The upper limit is determined by the laser linewidth. It is $6 \mathrm{~ns}^{-1}$ (corresponding to $1 \mathrm{GHz}$ width) for the high resolution scans, and $20 \mathrm{~ns}^{-1}$ for the broadband scan. The latter value was obtained by assuming that the laser bandwidth in broadband mode is wider than $4 \mathrm{GHz}$.

For each state, the measurements were performed at three different atomic density points in $(0.2-2) \times 10^{21} \mathrm{~cm}^{-3}$, and no effects were observed for all the cases.

\section{RESULTS AND DISCUSSION}

Figure 2 and Table I show the results of our decay rate measurements for all the 18 (including eight already published) states. Theoretical values by Korobov and Shimamura [13], Kartavtsev with co-workers [11], and the latest calculations by Korobov [7] and Kino [9] are also shown.

Most of the experimental decay rates are roughly in agreement with the calculations. By using these results, we were able to expand the recent precise experiment-theory comparison of the level energies [4] to the complex plane. In Fig. 3, the complex energy, defined as Eq. (1), was compared with values from two theories, [7] and [9]. For the imaginary 
TABLE I. Experimental decay rates and theoretically calculated Auger decay rates in Refs. $[7,9,11,13]$ in $\mathrm{s}^{-1} . L$ is the minimum transition multipolarity of Auger decay. All the data including past measurements in Refs. $[14,15]$ are shown. For the $(39,35)$ and $(37,34)$ states of $\bar{p}^{4} \mathrm{He}^{+}$, the radiative decay is dominant and the experimental decay rates cannot directly be compared with theoretical Auger rates. Some of the experimental values are not directly determined and only the possible ranges are shown (see the case of Sec. II C).

\begin{tabular}{|c|c|c|c|c|c|c|c|c|}
\hline State & $L$ & Experimental values & & KRB-SMM [13] & KRT [11] & KRB [7] & KNO $[9,24]$ & \\
\hline \multicolumn{9}{|l|}{$\bar{p}^{4} \mathrm{He}^{+}$} \\
\hline$(38,33)$ & 2 & $(3.85 \pm 0.26) \times 10^{11}$ & {$[15]$} & $3.1 \times 10^{11}$ & $3 \times 10^{11}$ & $3.07 \times 10^{11}$ & $6.45 \times 10^{11}$ & \\
\hline$(36,32)$ & 2 & $(6.35 \pm 0.65) \times 10^{11}$ & & $5.8 \times 10^{11}$ & $6.1 \times 10^{11}$ & $5.59 \times 10^{11}$ & $5.95 \times 10^{11}$ & \\
\hline$(39,34)$ & 3 & $(0.1-6) \times 10^{9}$ & & $7.7 \times 10^{8}$ & $1 \times 10^{9}$ & $8.21 \times 10^{8}$ & & \\
\hline$(38,34)$ & 3 & $(1.11 \pm 0.07) \times 10^{8}$ & [15] & $1.3 \times 10^{8}$ & $1.4 \times 10^{8}$ & $1.344 \times 10^{8}$ & & \\
\hline$(37,33)$ & 3 & $(1.11 \pm 0.16) \times 10^{11}$ & [15] & $5.7 \times 10^{9}$ & $3 \times 10^{10}$ & $4.21 \times 10^{10}$ & $4.38 \times 10^{10}$ & \\
\hline$(36,33)$ & 3 & $(2.2 \pm 0.8) \times 10^{8}$ & [14] & $2.4 \times 10^{8}$ & & $2.42 \times 10^{8}$ & $3.31 \times 10^{8}$ & \\
\hline$(35,32)$ & 3 & $(1.10 \pm 0.53) \times 10^{11}$ & & $3.7 \times 10^{9}$ & $7.5 \times 10^{9}$ & $5.76 \times 10^{9}$ & $6.04 \times 10^{9}$ & \\
\hline$(34,32)$ & 3 & $(2.36 \pm 0.47) \times 10^{8}$ & & $2.2 \times 10^{8}$ & $2.3 \times 10^{8}$ & $2.260 \times 10^{8}$ & $2.5 \times 10^{8}$ & \\
\hline$(39,35)$ & 4 (rad.) & $(6.5 \pm 0.2) \times 10^{5}$ & [14] & $7.0 \times 10^{4}$ & $8 \times 10^{4}$ & & & \\
\hline$(37,34)$ & 4 (rad.) & $(6.7 \pm 0.5) \times 10^{5}$ & [14] & $1.9 \times 10^{5}$ & & & & \\
\hline$(32,31)$ & 4 & $(1.17 \pm 0.12) \times 10^{8}$ & [15] & $6.1 \times 10^{5}$ & & $4.2 \times 10^{4}$ & & \\
\hline \multicolumn{9}{|l|}{$\bar{p}^{3} \mathrm{He}^{+}$} \\
\hline$(37,32)$ & 2 & $(1.24 \pm 0.26) \times 10^{12}$ & & $6.6 \times 10^{11}$ & & $6.71 \times 10^{11}$ & $6.74 \times 10^{11}$ & \\
\hline$(37,33)$ & 3 & $(0.1-6) \times 10^{9}$ & & $3.3 \times 10^{8}$ & $3.8 \times 10^{8}$ & $3.52 \times 10^{8}$ & & \\
\hline$(36,32)$ & 3 & $(0.1-20) \times 10^{9}$ & & $1.1 \times 10^{12}$ & $1.2 \times 10^{8}$ & $4.8 \times 10^{8}$ & $7.0 \times 10^{8}$ & \\
\hline$(35,32)$ & 3 & $(0.1-6) \times 10^{9}$ & & $6.8 \times 10^{8}$ & $7 \times 10^{8}$ & $6.76 \times 10^{8}$ & $4.1 \times 10^{8}$ & \\
\hline$(34,31)$ & 3 & $(1.84 \pm 0.65) \times 10^{10}$ & & $3.5 \times 10^{10}$ & & $6.7 \times 10^{7}$ & $5.4 \times 10^{7}$ & {$[24]$} \\
\hline$(33,31)$ & 3 & $(0.1-6) \times 10^{9}$ & & $6.9 \times 10^{8}$ & $6.9 \times 10^{8}$ & $6.85 \times 10^{8}$ & $7.4 \times 10^{8}$ & \\
\hline$(31,30)$ & 3 & $(0.1-6) \times 10^{9}$ & & & & $2.76 \times 10^{8}$ & $2.5 \times 10^{8}$ & \\
\hline
\end{tabular}

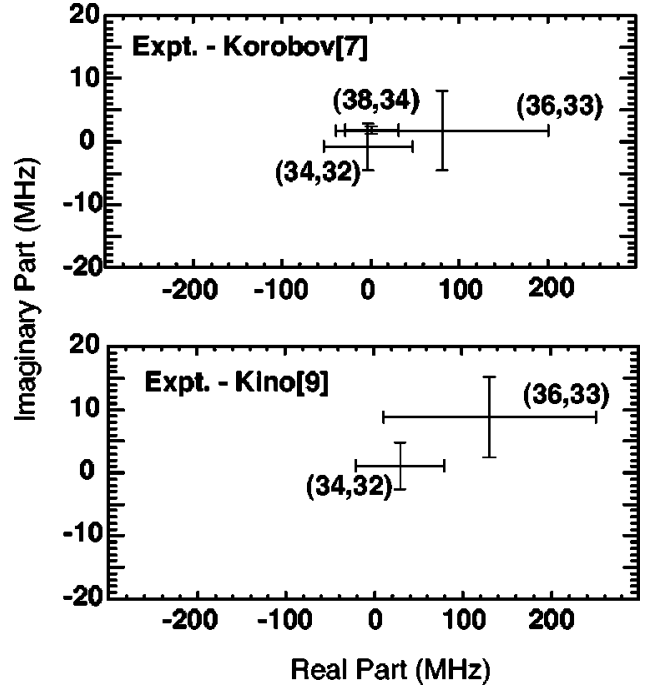

FIG. 3. Precise comparison of the complex energies of three $\bar{p}^{4} \mathrm{He}^{+}$states. The difference of the experimental values from the two theories in Refs. [7,9] are plotted in megahertz. The imaginary part plotted here is the half Auger width with negative sign, $-\frac{1}{2}\left(\gamma_{A} / 2 \pi\right)$. The values used for the comparison of the real part are the transition frequencies from metastable states, where the theoretical uncertainty should be very small. part (i.e., the half Auger width) of the three $\bar{p}^{4} \mathrm{He}^{+}$states presented in Fig.3, no discrepancy greater than $10 \mathrm{MHz}$, was found. This limit is absolutely even more precise than that obtained for the real part (i.e., the level energy).

A noteworthy state is the $(36,32)$ of $\bar{p}^{3} \mathrm{He}^{+}$. Although an $a b$ initio calculation [13] predicted a large anomaly, its decay rate was found to be nonanomalous. Another calculation [11] was done by introducing continuum-coupled wave functions explicitly in the equation of motion, and resulted in a nonanomalous value. After the present experiment was finished Korobov presented the results of a new calculation with the CCR method [7]. These are in good agreement with our experiment, suggesting that the coupling with the continuum is well represented by this method.

As for the latest results obtained in this work, two anomalous candidates have been found. They were the $(35,32)$ state of $\bar{p}^{4} \mathrm{He}^{+}$and the $(34,31)$ state of $\bar{p}^{3} \mathrm{He}^{+}$, both of which have much faster decay rates than the typical values expected by Eq. (2). Theoretical Auger rates are also faster than the typical rates for the $(35,32)$ state of $\bar{p}^{4} \mathrm{He}^{+}$, but there are large discrepancies between the calculations for the $(34,31)$ state of $\bar{p}^{3} \mathrm{He}^{+}$. Korobov [13] and Kino [9] both obtained anomalous values in the old calculation, but their latest results (Refs. [7] and [24], respectively) are nonanomalous.

The reason for these anomalies are possibly the same as the case of the $(37,33)$ state of $\bar{p}^{4} \mathrm{He}^{+}[15]$, the rate of which is supposed to be increased by a state mixing with an 
electron-excited configuration having a nearby level energy. Since an excited electron is far away from the nucleus compared to the distance of the other two particles, the energy of the electron-excited states can be approximated by the following formula $[11,15]$ :

$$
E=-\frac{m_{\bar{p}} m_{\alpha}}{m_{\bar{p}}+m_{\alpha}} \frac{Z^{2}}{2 n_{\bar{p}}^{2}}-\frac{1}{2 n_{e}^{2}},
$$

in atomic units. Here $Z=2$ is the atomic number of helium. As are illustrated in Fig. 4, the configurations $(31,30)_{\bar{p}}$ $\otimes(3 d)_{e}$ for the $(35,32)$ state of $\bar{p}^{4} \mathrm{He}^{+}$, and $(30,29)_{\bar{p}}$ $\otimes(3 d)_{e}$ for the $(34,31)$ state of $\bar{p}^{3} \mathrm{He}^{+}$can be the reason for the anomalies in the same way. For the latter case, the possibility of the influence by an electron-excited state was pointed out by Kartavtsev [11].

There is still possibility that the rate enhancements were caused by collisions, since we do not know the experimental decay rates of the atom isolated in vacuum. In any case, studying these states both experimentally and theoretically should be a good test for understanding the continuumcoupled three-body system.

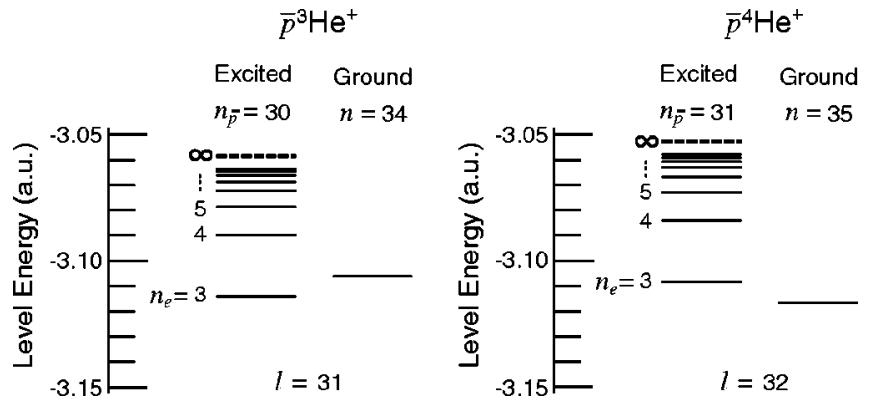

FIG. 4. Energy diagram of the ground-electron states $(34,31)$ of $\bar{p}^{3} \mathrm{He}^{+}$and $(35,32)$ of $\bar{p}^{4} \mathrm{He}^{+}$, and their neighbor electron-excited states. The energy values of the ground-electron states were obtained by Ref. [5], and those of electron-excited states were obtained by Eq. (4).

\section{ACKNOWLEDGMENTS}

The authors are grateful to the CERN PS division for their help; V. I. Korobov, D. D. Bakalov, and Y. Kino for useful discussions. This work was supported by the Grant-in-Aid for Specially Promoted Research (Grant No. 15002005) of MEXT, Japan, and the Hungarian Scientific Research Fund (Grant Nos. OTKA T046095 and TeT-Jap-4/00).
[1] M. Iwasaki, S. N. Nakamura, K. Shigaki, Y. Shimizu, H. Tamura, T. Ishikawa, R. S. Hayano, E. Takada, E. Widmann, H. Outa, M. Aoki, P. Kitching, and T. Yamazaki, Phys. Rev. Lett. 67, 1246 (1991).

[2] T. Yamazaki, E. Widmann, R. S. Hayano, M. Iwasaki, S. N. Nakamura, K. Shigaki, F. J. Hartmann, H. Daniel, T. von Egidy, P. Hofmann, Y.-S. Kim, and J. Eades, Nature (London) 361, 238 (1993).

[3] T. Yamazaki, N. Morita, R. S. Hayano, E. Widmann, and J. Eades, Phys. Rep. 366, 183 (2002).

[4] M. Hori, J. Eades, R. S. Hayano, T. Ishikawa, W. Pirkl, E. Widmann, H. Yamaguchi, H. A. Torii, B. Juhász, D. Horváth, and T. Yamazaki, Phys. Rev. Lett. 91, 123401 (2003).

[5] V. I. Korobov, Phys. Rev. A 54, R1749 (1996).

[6] V. I. Korobov and D. D. Bakalov, Phys. Rev. Lett. 79, 3379 (1997).

[7] V. I. Korobov, Phys. Rev. A 67, 062501 (2003); 68, 019902(E) (2003).

[8] Y. Kino, N. Yamanaka, M. Kamimura, P. Froelich, and H. Kudo, Hyperfine Interact. 138, 179 (2001).

[9] Y. Kino, M. Kamimura, and H. Kudo, Nucl. Instrum. Methods Phys. Res. B 214, 84 (2004).

[10] J. E. Russell, Phys. Rev. A 1, 742 (1970).

[11] O. Kartavtsev, D. Monakhov, and S. Fedotov, Phys. Rev. A 61, 062507 (2000); 63, 019901(E) (2000).

[12] T. Yamazaki and K. Ohtsuki, Phys. Rev. A 45, 7782 (1992); K. Ohtsuki (unpublished).

[13] V. I. Korobov and I. Shimamura, Phys. Rev. A 56, 4587 (1997).

[14] M. Hori, H. A. Torii, R. S. Hayano, T. Ishikawa, F. E. Maas, H. Tamura, B. Ketzer, F. J. Hartmann, R. Pohl, C. Maierl, M.
Hasinoff, T. von Egidy, M. Kumakura, N. Morita, I. Sugai, D. Horváth, E. Widmann, J. Eades, and T. Yamazaki, Phys. Rev. A 57, 1698 (1998); 58, 1612(E) (1998).

[15] H. Yamaguchi, T. Ishikawa, J. Sakaguchi, E. Widmann, J. Eades, R. S. Hayano, M. Hori, H. A. Torii, B. Juhász, D. Horváth, and T. Yamazaki, Phys. Rev. A 66, 022504 (2002).

[16] A. M. Lombardi, W. Pirkl, and Y. Bylinsky, in Proceedings of the 2001 Particle Accelerator Conference, Chicago (IEEE, Piscataway, NJ, 2001), pp. 585-587.

[17] M. Hori, J. Eades, E. Widmann, H. Yamaguchi, J. Sakaguchi, T.Ishikawa, R. S. Hayano, H. A. Torii, B. Juhász, D. Horváth, and T. Yamazaki, Phys. Rev. Lett. 87, 093401 (2001).

[18] N. Morita, K. Ohtsuki, and T. Yamazaki, Nucl. Instrum. Methods Phys. Res. A 330, 439 (1993).

[19] N. Morita, M. Kumakura, T. Yamazaki, E. Widmann, H. Masuda, I. Sugai, R. S. Hayano, F. E. Maas, H. A. Torii, F. J. Hartmann, H. Daniel, T. von Egidy, B. Ketzer, W. Müller, W. Schmid, D. Horváth, and J. Eades, Phys. Rev. Lett. 72, 1180 (1994).

[20] M.Hori, K.Yamashita, R. S. Hayano, and T. Yamazaki, Nucl. Instrum. Methods Phys. Res. A 496, 102 (2002).

[21] E. Widmann, J. Eades, T. Yamazaki, H. A. Torii, R. S. Hayano, M. Hori, T. Ishikawa, M. Kumakura, N. Morita, I. Sugai, F. Hartmann, T. von Egidy, B. Ketzer, C. Maierl, R. Pohl, and D. Horváth, Phys. Lett. B 404, 15 (1997).

[22] E. Widmann, J. Eades, T. Ishikawa, J. Sakaguchi, T. Tasaki, H. Yamaguchi, R. S. Hayano, M. Hori, H. A. Torii, B. Juhász, D. Horváth, and T. Yamazaki, Phys. Rev. Lett. 89, 243402 (2002).

[23] D. Bakalov and V. I. Korobov, Phys. Rev. A 57, 1662 (1998).

[24] Y. Kino (private communication). 